Service social

\title{
De l’aide sociale à la sécurité du revenu au Québec
}

\section{Denis Fortin}

Volume 39, numéro 2, 1990

Les problèmes sociaux

URI : https://id.erudit.org/iderudit/706483ar

DOI : https://doi.org/10.7202/706483ar

Aller au sommaire du numéro

Éditeur(s)

École de service social de l'Université Laval

ISSN

1708-1734 (numérique)

Découvrir la revue

Citer cet article

Fortin, D. (1990). De l'aide sociale à la sécurité du revenu au Québec. Service social, 39(2), 159-174. https://doi.org/10.7202/706483ar

\section{Résumé de l'article}

Procédant à une analyse critique de la récente réforme de l'aide sociale au Québec, l'auteur est amené à faire un lien entre problèmes sociaux et politiques sociales. Après avoir rappelé le contexte socio-économique (crise économique au début des années 1980, crise de l'État-Providence, etc.), il estime que cette réforme s'inscrit dans un "virage social », marqué au coin d'un "néo-conservatisme ", ou d'un " Reaganisme social », d'où son caractère punitif vis-à-vis les assistés sociaux définis comme « une bande de paresseux et de fraudeurs ». Mais après avoir décrit et commenté les principaux éléments de cette réforme, l'auteur estime que cette réforme risque de produire, par la fameuse alchimie des effets pervers, des résultats contraires à ceux escomptés, soit « l'amplification de l'appauvrissement des bénéficiaires et de l'oppression sociale dont ils sont déjà victimes ». Finalement, D. Fortin montre comment cette réforme s'inscrit au coeur d'un nouveau projet de société.
Ce document est protégé par la loi sur le droit d'auteur. L'utilisation des services d’Érudit (y compris la reproduction) est assujettie à sa politique d'utilisation que vous pouvez consulter en ligne.

https://apropos.erudit.org/fr/usagers/politique-dutilisation/ 


\section{COMMENTAIRES \\ ET DOCUMENTS}

Denis Fortin, professeur à l'École de service social, Université Laval.

\section{De l'aide sociale à la sécurité du revenu au Québec}

Denis Fortin

Le présent article vise à brosser un tableau sommaire des principales données qui caractérisent la nouvelle Loi (37) sur la sécurité du revenu au Québec, adoptée par l'Assemblée nationale le 22 décembre 1988 et mise en application à compter du $1^{\text {er }}$ août 1989. La préparation de ce contenu fait suite à la publication, en 1988, d'un ouvrage portant sur cette question et ayant pour titre : Riches contre pauvres : deux poids, deux mesures. Au passage... de l'État-Providence à l'État-Provigo ! Il incorpore également certains éléments clés préparés aux fins d'une communication présentée en septembre 1989 dans le cadre des assises d'un colloque tenu à Montréal sur le thème "Santé mentale et pauvreté : un silence troublant" ".

En conséquence et à l'instar des productions auxquelles il est fait référence précédemment, cet article se propose d'intégrer tant la dimension strictement descriptive de la question traitée que la dimension analytique, laquelle a pour effet de situer notre démarche dans une perspective à la fois historique et critique, perspective tout à fait essentielle si nous ambitionnons véritablement de repérer, derrière le discours officiel et certaines apparences, le sens profond, la portée et l'impact concret de cette importante législation dans la présente conjoncture. 


\section{La " crise " : occasion d'un virage social radical... !}

Reportons-nous au tournant des années 1980 : la crise économique bat son plein. Elle contraindra les tenants du capital à une restructuration d'ensemble du mode de production à l'échelle de l'économie-monde, au Canada et au Québec en particulier, dans un sens que l'on apprendra progressivement à désigner par le concept de "néo-libéralisme".

$C^{\prime}$ est dans ce contexte que l'État-Providence, après avoir joué le rôle de bouée de sauvetage du système capitaliste lors de la « Grande crise des années $1930 "$, constituera désormais, dans ce nouveau contexte, la cible privilégiée des attaques menées sans merci contre l'État et le «social». Au cœur de la politique sociale, c'est le programme de l'aide sociale, mis en place en décembre 1969, qui sera l'objet des critiques les plus dures et insistantes et des remises en cause les plus fondamentales. Mais des questions se posent à ce propos!

Pourquoi cette réforme d'abord et pourquoi lui avoir donné priorité ? Quels sont les principes qui en ont déterminé l'orientation principale et sa traduction législative sous la forme de l'actuelle Loi sur la sécurité du revenu ? Quels sont les impacts prévisibles de la mise en application de cette loi, compte tenu de ce qu'elle vise et de la pratique partielle récente ( $1^{\mathrm{er}}$ août 1989) à cet égard ? Des questions importantes, essentielles s'il en est, puisqu'elles concernent directement le sort désormais réservé à des centaines de milliers de citoyennes et citoyens du Québec qui comptent parmi les plus démunis. Parmi eux, nombreux sont ceux pour qui les problèmes liés à la santé mentale ne sont pas étrangers, vu les conditions de vie particulièrement difficiles qui leur sont réservées. En outre, I'aide sociale constitue sans contredit le lieu d'accueil principal pour les personnes souffrant de déficience intellectuelle ou mentale et qui font les frais de la politique de désinstitutionnalisation telle que pratiquée présentement, c'est-à-dire en l'absence d'un réservoir suffisant de ressources alternatives appropriées.

\section{Un virage social radical... ! Par qui ? Pour qui ? Contre qui ?}

Ces questions plus fondamentales soulignent toute l'importance de la présente réflexion, par rapport à l'aide sociale entre autres. Mais pour qu'un virage social radical puisse être pris et que le vent tourne brusquement dans le sens de l'État-Providence à l'État-Provigo, encore fallait-il trouver un alibi qui soit suffisant ! 
Le déficit budgétaire de l'État comblera cette exigence stratégique pour le Bloc au pouvoir. II produira même davantage, soit la mise en place des conditions propices à l'émergence d'un véritable climat de panique, de psychose quasi obsessionnelle, tant au niveau des élites économiques et politiques et des intellectuels qu'au niveau des différents médias et de la population d'une manière plus générale.

Au cœur de cette panique pouvaient désormais prendre solidement ancrage les valeurs d'un "néo-conservatisme " inspiré du grand courant dominant du thatchérisme reaganien. Et, pour parvenir à l'objectif visé, le "responsable de tous nos maux " devait être rapidement repéré, identifié et désigné, puis exposé sur la place publique. On voulait ainsi calmer cette peur et cette confusion savamment entretenues par ailleurs dans l'esprit d'une majorité, de même que chez bon nombre d'intervenants et intervenantes et de spécialistes du champ de la politique sociale, en l'occurrence.

Bref, ce faisant, le tour était joué : " coupables ou pas coupables..., les assistés sociaux doivent payer !», et les premiers. Le 22 décembre 1988, la Loi sur la sécurité du revenu au Québec, incorporant la réforme Paradis-Bourbeau de l'aide sociale, était finalement adoptée par l'Assemblée nationale, dans un climat de relative précipitation et de confusion. Dix-neuf années, presque jour pour jour, après l'adoption du fameux «Bill 26 » par le Parlement en 1969.

\section{Les assistés sociaux : une bande de " paresseux et de fraudeurs " !}

Afin de s'assurer de la légitimité sociale nécessaire à l'opération de chirurgie déjà prévue pour ce secteur, il était impératif pour le Gouvernement de discréditer au maximum la population visée par cette réforme stratégique, en l'associant à une "vulgaire bande de fraudeurs et de paresseux ». Pour parvenir à cet objectif, encore fallait-il cueillir quelques preuves hors de tout doute et procéder soigneusement à leur étalage en public, pour ajouter au discrédit visé. À cette fin, 150 enquêteurs spéciaux sont embauchés au coût de $9000000 \$$ et mis en poste d'attaque à compter de mai 1986. Ils ont pour mission de pourchasser par tous les moyens les assistés sociaux, les femmes en particulier, et d'identifier à tout prix "fraudeurs et paresseux qui vivent indûment au crochet de l'État». Ceux-ci deviennent vite célèbres : les "Bouboumacoutes».

Depuis la mise en application de la nouvelle loi, cette offensive répressive ne s'est guère estompée, bien au contraire. Elle se poursuit de plus belle, emprunte d'autres moyens, vise d'autres cibles et tend 
même à s'amplifier, du moins par rapport à certaines catégories de bénéficiaires, en l'occurrence les personnes dites «inaptes au travail ", ce qui est nouveau. De toute évidence, les derniers chapitres de cette aventure sont loin d'être terminés ${ }^{2}$.

Par ailleurs, le caractère particulièrement odieux de cette offensive sera l'occasion d'un important développement des forces d'opposition aux orientations de cette réforme. Au fil d'une longue lutte de résistance politique, quelque 1700 groupes à travers tout le Québec établiront alliance et accorderont leur appui aux revendications du Front commun des personnes assistées sociales, fer de lance de cette même lutte. Un épisode tout à fait unique, sans précédent dans les annales de l'histoire des luttes populaires de résistance au Québec. Pourtant, et malgré tout cela...!

\section{Une réforme reaganienne et punitive... ou « de l'aide sociale (1969) à la sécurité du revenu (1989) " !}

Si l'intitulé de cette rubrique peut paraître contradictoire dans l'esprit de certains, dans la réalité il n'en est rien. L'analyse critique de la nouvelle politique sur la sécurité du revenu nous révèle en effet sans aucune équivoque son caractère régressiste. Qu'en est-il au juste?

La Loi sur la sécurité du revenu au Québec procède à la transformation du système antérieur de l'aide sociale (loi 26) en trois programmes distincts $^{3}$ :

— SOUTIEN FINANCIER, réservé aux bénéficiaires de l'aide sociale « inaptes au travail »;

- APTE, pour Actions positives pour le travail et l'emploi, destiné aux bénéficiaires de l'aide sociale "aptes au travail » et répartis en quatre grandes catégories :

- disponibles

- non-disponibles

- participants

- non-participants;

- APPORT, pour Aide aux parents pour leurs revenus de travail, destiné aux familles de petits salariés avec enfants à charge.

Le tableau-synthèse qui suit vise précisément à clarifier, de manière sommaire, les orientations principales et les grandes composantes de cette nouvelle politique sur la sécurité du revenu au Québec (voir tableau 1). Ce qu'il importe de savoir, c'est que toute l'économie de la loi 37 repose sur une notion clé : "l'employabilité » 
des bénéficiaires, ou encore l'aptitude ou l'inaptitude au travail au sens déjà connu de ces termes.

La loi définit ainsi ces notions clés. L'employabilité réfère à une personne qui a la capacité de travailler et de subvenir à ses besoins. En contrepartie, la non-employabilité désigne une personne qui ne peut travailler pour subvenir à ses besoins et à ceux de sa famille pour les raisons suivantes : déficience, maladie physique ou mentale; cet état est permanent ou de longue durée (12 mois et plus); cet état est constaté par un rapport médical.

$C^{\prime}$ est donc sur cette base première, l'employabilité, que reposent toute la structure et les orientations de cette nouvelle politique sur la sécurité du revenu. Ainsi, dans le cas des personnes "non employables", la loi prévoit le maintien d'un programme d'assistance, dit SOUTIEN FINANCIER, et la révision très légèrement à la hausse du barème des prestations, pour lequel une indexation automatique est

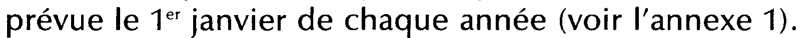

Le programme APTE, pour sa part, généralise à toutes les personnes assistées sociales classées "employables " I'application des mesures de réinsertion sociale déjà mises sur pied en 1983-1984 et complétées depuis par de nouvelles mesures. Et, depuis le $1^{\text {er }}$ août 1989 , suite à une brève période transitoire dans leur cas et s'ils sont toujours admissibles par rapport aux nouvelles normes, les bénéficiaires de moins de trente ans touchent désormais le même niveau de prestation que les plus âgés, ce qui n'était pas le cas antérieurement. Par ailleurs, pour l'ensemble de cette catégorie de bénéficiaires (c'est-à-dire les " aptes"), les prestations versées sont réduites à un strict minimum, à moins qu'ils acceptent de participer à l'un ou l'autre des volets du programme APTE, qui vise explicitement à développer les qualités minimales "d'employabilité " des personnes concernées et, surtout, à les resituer le plus rapidement possible sur le marché du travail. De plus, la loi ne prévoit pas l'indexation automatique des différents barèmes de prestations dans le cas du programme APTE.

En ce qui concerne la panoplie des mesures prévues dans le cadre de ce programme aux fins du " développement de l'employabilité et de l'aide à l'emploi », elle s'établit comme suit :

Mesures antérieures à la loi 37 :

_ rattrapage scolaire,

- retour aux études postsecondaires pour les chefs de famille monoparentale,

- travaux communautaires,

- bon d'emploi plus,

- services externes de main-d'œuvre. 


\section{La politique de sécurité du revenu au Québec} Synthèse de la loi 37

\begin{tabular}{|c|c|c|}
\hline Soutien financier & $\begin{array}{l}\text { APTE } \\
\text { Actions positives pour le travail et l'emploi }\end{array}$ & $\begin{array}{l}\text { APPORT } \\
\text { Aide aux parents pour } \\
\text { revenus de travail }\end{array}$ \\
\hline $\begin{array}{l}\text { OBIECTIFS DU PROGRAMME } \\
\text { - Répondre plus généreusement } \\
\text { qu'auparavant aux besoins des } \\
\text { personnes incapables de } \\
\text { travailler, soit les « inaptes au } \\
\text { travail ". } \\
\text { - Simplifier les modalités admi- } \\
\text { nistratives entourant le verse- } \\
\text { ment de leurs prestations. }\end{array}$ & $\begin{array}{l}\text { OBJECTIFS DU PROGRAMME } \\
\text { - "Encourager " l'intégration ou la réintégration au marché du travail } \\
\text { des bénéficiaires de l'aide sociale dits " aptes au travail ». } \\
\text { - À cette fin, trois moyens principaux sont prévus : } \\
\text { 1- Une aide financière couvrant une partie des besoins de base. Pour } \\
\text { être « incitatif au travail », le montant de ces prestations maintient } \\
\text { un écart significatif entre le revenu disponible des familles dont le } \\
\text { chef travaille au salaire minimum et celui des clients de l'aide } \\
\text { sociale dit « employables ". D'autre part, le montant des presta- } \\
\text { tions et le niveau des gains de travail exclus du calcul des ressources } \\
\text { sont désormais fonction de la disponibilité des clients et de la } \\
\text { participation ou de la non-participation aux mesures d'aide à l'in- } \\
\text { tégration au marché du travail. } \\
\text { 2- Une aide à l'emploi (ex. Bon d'emploi plus, conversion des presta- } \\
\text { tions d'aide sociale "Grant Diversion "). } \\
\text { 3- Un ensemble de mesures d'aide au développement de l'em- } \\
\text { ployabilité (ex. services d'orientation, de formation, travaux com- } \\
\text { munautaires, stage en milieu de travail, etc.). }\end{array}$ & $\begin{array}{l}\text { OBJECTIFS DU PROGRAMME } \\
\text { - Soutenir le revenu des } \\
\text { travailleurs à faibles revenus. Ce } \\
\text { faisant, l'intention poursuivie } \\
\text { est double : } \\
\text { - inciter les travailleurs qui ont } \\
\text { des revenus modestes et des } \\
\text { enfants à charge à rester sur le } \\
\text { marché du travail; } \\
\text { - inciter les personnes qui re- } \\
\text { çoivent des prestations d'as- } \\
\text { surance-chômage ou d'aide } \\
\text { sociale, et qui ont des enfants } \\
\text { à charge, à entrer sur le mar- } \\
\text { ché du travail. }\end{array}$ \\
\hline
\end{tabular}


POPULATION VISÉE

- Personnes qui présentent des contraintes sévères à l'emploi (c'est-à-dire toute personne dont l'état physique ou mental est de façon significative altéré ou déficient, pour une durée permanente ou indéfinie, et qui est empêchée, cela étant, de subvenir à ses besoins).

- Également toute famille dont I'un des conjoints est dans cet état.

- En mars 1988 : 94,000 ménages de cette catégorie, ou 26,3\% de la clientèle de l'aide sociale.

\section{BAREME}

- Équivalent au $10 \%$ des ménages de travailleurs qui ont les plus faibles revenus au pays (selon Statistiques Canada).

\section{POPULATION VISÉE}

- Toute personne APTE AU TRAVAIL et bénéficiaire de l'aide sociale.

- En mars 1988, cette population comptait au total 264,000 chefs de ménage vivant de l'aide sociale, et répartis comme suit :

- 183,000 considérés comme employables et disponibles, soit 51,1 \% de la clientèle totale de l'aide sociale

- 81,000 considérés comme employables et non disponibles, soit $22,6 \%$ de la clientèle totale.

\section{POPULATION VISÉE}

- Travailleurs à faibles revenus qui :

- ont au moins un enfant à charge;

- gagnent des revenus de travail d'au moins 150 \$au cours d'un mois donné et touchent moins de $300 \$$ des organismes gouvernementaux au cours de ce mois.

\section{BARÈME}

\section{BARÈME}

- Quatre barèmes de besoins sont établis pour correspondre aux quatre grandes catégories de bénéficiaires de ce programme, à savoir :

- Barème de non-disponibilité

- Barème de disponibilité

- Barème de non-participation

- Barème de participation

- Un barème mixte est également prévu pour le cas où les deux adultes d'une même famille ne font pas partie de la même catégorie de bénéficiaires. revenu familial est versée sur une base mensuelle.

- Le calcul de celle-ci tient compte de plusieurs facteurs (v.g. revenus, actifs, frais de garde, etc.)

- Mais dans tous les cas, le montant de cette " aide personnalisée " se situe très en deçà des seuils de pauvreté.

SOURCE : Tableau construit à partir d'informations tirées de La sécurité du revenu. En bref, Ministère de la Main-d'œuvre et de la Sécurité du revenu, Québec, 1989, 30 pages. 
Nouvelles mesures introduites depuis :

- reconnaissance des activités de développement de l'employabilité (RADE),

- conversion des prestations en vue de favoriser l'intégration au marché du travail ("Grant Diversion»).

Par défaut de pouvoir expliciter ici le contenu de chacune de ces mesures, retenons simplement qu'avec la loi 37 , tous les bénéficiaires de 18 à 65 ans jugés aptes au travail devront s'inscrire à une activité de réinsertion sous peine de voir leur prestation réduite au strict minimum (voir les annexes 2 et 3 ).

Cette législation introduit également un certain nombre d'autres « notions nouvelles » dont l'effet principal consiste à restreindre la couverture de la loi, tout en ajoutant aux contraintes déjà exercées à l'encontre des bénéficiaires. Nous pensons entre autres à la notion qui concerne la "contribution parentale obligatoire", qui sert à établir l'inadmissibilité partielle ou totale de plusieurs milliers "d'adultes de 18 ans ou plus qui n'ont pas encore affirmé leur indépendance ", au sens de l'article 633 du Code civil. Ou encore, à la notion dite de "non-disponibilité » (versus "disponibilité ») pour des bénéficiaires jugés aptes au travail qui, pour certaines raisons (ex. cas de grossesse, personne malade pendant moins de douze mois, adulte ayant un enfant à charge qui ne fréquente pas l'école, ou adulte de 55 ans et plus), ne peuvent participer aux différentes mesures de réinsertion sociale et qui sont pénalisés, ce faisant, du seul fait de leur état. S'ajoutent finalement les notions de "participant " et "non-participant " aux mesures de réinsertion, celle de "conjoint", celle de "partage du logement », etc., toutes notions dont la fonction consiste à particulariser la masse des bénéficiaires, à la diviser en segments et à appliquer à chacun d'eux un traitement normatif approprié. L'impact recherché est de maximiser le lot des contraintes à subir, tout en réduisant la contrepartie des bénéfices matériels possibles et, surtout, la base des droits sociaux ainsi reconnus, ce qui est encore plus fondamental.

De ce tour d'horizon par trop sommaire de la nouvelle politique de sécurité du revenu au Québec, nous pouvons retenir que pour chacun des trois grands programmes constitutifs, comme pour chaque catégorie possible de bénéficiaires, tant le traitement normatif prévu que la structure des barèmes de prestations sont spécifiques, ce qui signifie, en bref, qu'ils sont multiples. D'où l'ampleur du défi, pour l'appareil gouvernemental, de la mise en application de cette politique et, pour les bénéficiaires concernés, de s'y retrouver et d'exiger respect de leurs droits.

Sans tenir compte des contrôles administratifs et répressifs additionnels qui ont également pris forme avec l'avènement de cette 
loi et qui se seront probablement accrus au cours de 1990, nous pouvons affirmer sans ambages que cette nouvelle politique de sécurité (ou encore d'insécurité) du revenu vient cristalliser en son sein plusieurs " nouveautés » qui ont contradictoirement pour effet de nous reporter en arrière, de plusieurs décennies, c'est-à-dire à l'étape de la préhistoire de la sécurité sociale, de la sécurité du revenu et de la politique sociale tout court.

En conséquence, nous pourrions dire, à propos des orientations et du contenu normatif qui définissent cette nouvelle politique, que I'héritage premier qui la caractérise se trouve nettement du côté $\mathrm{d}^{\prime}$ une certaine forme de reaganisme social. Les propensions "néolibérales " de cette politique sont à chercher principalement du côté d'une idéologie profondément conservatrice qui, dans le contexte actuel, pourrait même être qualifiée de réactionnaire. Non seulement y a-t-il la lettre de la loi, mais également et surtout l'esprit qui préside à sa mise en application et à sa gestion administrative et " politique ». Or, à cet égard, la pratique actuelle parle beaucoup plus éloquemment que tout ce que nous pourrions tenir comme discours...

\section{Sécurité du revenu ou appauvrissement et oppression?}

Dans la foulée de cette logique, il n'est pas spécialement étonnant de constater que cette nouvelle politique, loin de donner forme au discours initial qui l'a engendrée à savoir « la mise en œuvre d'une nouvelle approche plus efficace en matière de lutte contre la pauvreté ", risque plutôt de réaliser l'inverse : l'amplification de l'appauvrissement des bénéficiaires et de l'oppression sociale dont ils sont déjà victimes de par leur état et leurs conditions de vie quotidiennes. Les données du tableau 2 tendent à accréditer cette dernière position plutôt que les grands énoncés humanistes du discours officiel tenu à ce propos tout au long de l'opération (voir tableau 2). Comme quoi, par un vice du discours, la pratique devant lui correspondre peut devenir parfaitement contradictoire et même produire son inversion pure et simple. C'est ainsi, par exemple, que le concept de "sécurité du revenu " chez les uns peut se traduire pour d'autres par "l'insécurité quotidienne", la peur, l'anxiété, l'autodévalorisation, le défaitisme, l'impuissance et, surtout, la privation et l'exclusion, bref, toutes situations qui spécifient et donnent sens de réalité au concept d'oppression. 
TABLEAU 2

Aide sociale et pauvreté

Une mesure du DEGRÉ DE PAUVRETÉ chez les assistés sociaux du Québec

\begin{tabular}{|c|c|c|c|c|c|c|c|c|}
\hline \multirow{4}{*}{\begin{tabular}{|l} 
Catégorie \\
Bénéficiaires \\
Nombre
\end{tabular}} & \multicolumn{5}{|c|}{ Barèmes de l'aide sociale 1989} & \multirow{4}{*}{$\begin{array}{c}\begin{array}{c}\text { Seuils } \\
\text { de }\end{array} \\
\text { pauvreté } \\
1989\end{array}$} & & \\
\hline & \multirow{3}{*}{$\begin{array}{l}\text { Soutien } \\
\text { financier } \\
\text { " inapte " }\end{array}$} & \multicolumn{4}{|c|}{ "Apte $" . .$. au travail } & & \multirow{2}{*}{\multicolumn{2}{|c|}{ Déficit budgétaire }} \\
\hline & & \multirow{2}{*}{$\begin{array}{c}\text { Non } \\
\text { disponible }\end{array}$} & \multirow[b]{2}{*}{ Disponible } & \multirow{2}{*}{$\begin{array}{c}\text { Non } \\
\text { participant }\end{array}$} & \multirow[b]{2}{*}{ Participant } & & & \\
\hline & & & & & & & Minimum & Maximum \\
\hline - 1 adulte & $* 7020 \$$ & $6084 \$$ & $5844 \$$ & $* * 5040 \$$ & $6240 \$$ & $11432 \$$ & $4412 \$$ & $6392 \$$ \\
\hline - 1 adulte, 1 enfant & * 9420 & 8280 & 8160 & ** 7440 & 8640 & 15074 & 5654 & 7634 \\
\hline - 1 adulte, 2 enfants et + & ${ }^{*} 10644$ & 9504 & 9384 & ** 8664 & 9864 & $20132+$ & 9488 & 11468 \\
\hline - 2 adultes & 10140 & 9672 & 9240 & $* * 7800$ & *10 200 & 15074 & 4874 & 7274 \\
\hline - 2 adultes, 1 enfant & 11520 & 10440 & 10620 & ** 9180 & *11 580 & 20132 & 8552 & 10952 \\
\hline - 2 adultes, 2 enfants et + & 12444 & 11304 & 11554 & **10 104 & *12 504 & $23266+$ & 10762 & 13162 \\
\hline
\end{tabular}

NOTE : Les seuils de pauvreté 1989 sont ceux établis par le Conseil national du bien-être social et correspondant aux agglomérations variant de 100000 à 499000 habitants.

* Catégories de bénéficiaires où le déficit budgétaire est minimum.

** Catégories de bénéficiaires où le déficit budgétaire est maximum. 


\section{La loi 37 : au cœur d'un nouveau projet de société}

Au terme de cette réflexion sur la loi 37, il faut comprendre que, derrière le discours officiel de légitimation, nécessaire en pareilles circonstances, le principe directeur de la réforme en cause consiste d'abord à réaliser à tout prix l'autosuffisance des bénéficiaires de l'aide sociale (les aptes au travail en particulier) et à accroître, en conséquence, les difficultés et contraintes qui sont liées au recours à la "sécurité du revenu ». D'une part, l'intention est de réduire la portion du budget de l'État affectée à cette fin, et d'autre part, de répondre aux exigences nouvelles de rentabilité et de performance des entreprises d'ici et qui, dans le présent contexte de l'Accord de libre échange, signifient la régression nécessaire des coûts de production, dont les salaires en priorité, et la mise en disponibilité d'un important bassin de main-d'œuvre bon marché susceptible de rendre l'opération possible. C'est à ce "Grand projet de société » que l'armée de réserve des assistés sociaux, la catégorie des " aptes au travail » en particulier, est systématiquement conviée depuis la mise en application de la Loi sur la sécurité du revenu.

Mais ces derniers ne sont pas les seuls à devoir partager le sort d'une condition sociale au bas de l'échelle. Car, nous le savons, cette réforme met également en place les mécanismes appropriés aux fins d'une consolidation des marchés périphériques de travail réservés aux "petits salariés", lesquels pourront désormais compléter une partie de l'insuffisance de leur revenu jusqu'à un seuil maximum, par le biais de certaines mesures financières compensatoires (programme APPORT). Il est par trop prévisible, cependant, que les petits salariés resteront, eux aussi, sans plus d'espoir de sortir, un jour..., des corridors aux parois étanches où ils sont finalement piégés. Les marchés périphériques de travail risquent fort de continuer d'être, dans le présent contexte, ces lieux privilégiés de culture et de reproduction de plus de $50 \%$ des couches de pauvreté au Canada et au Québec. Ce qui est actuellement le cas ne peut que se maintenir, sinon peut-être même progresser, avec la nouvelle loi, dans le sens d'une certaine détérioration de la situation.

En 1990 au Québec, et au terme de sept années de croissance économique continue qui nous annoncent une période de ralentissement, déjà amorcée par ailleurs et que viendra amplifier à coup sûr la mise en vigueur de la nouvelle TPS (taxe sur les produits et services) et les impacts à court terme résultant de l'Accord de libre échange, le taux de chômage officiel n'en continue pas moins d'osciller, de mois en mois, à quelque $10 \%$ de la population active.

Dans le cas de l'aide sociale (la nouvelle sécurité du revenu), il faut compter un taux qui varie autour de $9 \%$ du total de la population. 
Et, si nous ajoutons à tout cela la portion des personnes en mesure de travailler mais qui, par désespoir de cause, ne cherchent plus d'emploi, c'est à une personne sur cinq, soit $20 \%$ de l'ensemble de la population active potentielle, qu'il faut présentement établir le taux d'inactivité économique au Québec. Ce qui constitue déjà en soi une sorte de catastrophe sociale tout à fait inacceptable, intolérable même pour les personnes et les familles qui sont les premières visées par cette forme radicale d'exclusion sociale.

$Y$ a-t-il une lueur d'espoir pour ceux et celles qui choient présentement au sous-sol de la grande pyramide sociale? Quelle espérance... ? Entre 1959 et 1989, la portion des emplois à temps partiel et précaires est passée de $30 \%$ à plus de $50 \%$ de l'ensemble du bassin des emplois au pays. Or, depuis la récession économique de 1981-1982, la détérioration de cette situation, loin de s'atténuer, s'est considérablement accélérée. En effet, depuis cette date, la majorité des emplois créés sont de "piètre qualité, instables, précaires et massivement mal rémunérés, sinon à bas salaires strictement ", ce qui est le cas de plus de $50 \%$ de ces nouveaux emplois, dont le salaire annuel est inférieur à $20000 \$$ et, dans plus de $30 \%$ des cas, inférieur à $10000 \$$. Au cours de 1986-1987, année record sur ce point, plus de $80 \%$ des nouveaux emplois créés au pays étaient de cet ordre ${ }^{4}$.

Or, en l'absence d'une stratégie de développement économique et social articulée autour de l'objectif du plein emploi et liée organiquement à celui non moins important d'une juste rémunération salariale fondée, quant à elle, sur le principe de la décence et de l'équité, la nouvelle politique de sécurité du revenu est totalement impuissante à modifier un tant soit peu les données et contraintes multiples de la conjoncture tracée plus haut. Dans ces circonstances, cette politique ne peut engendrer d'autres effets que ceux liés à la détérioration accélérée de la condition sociale et économique plus générale des gens qui sont déjà au bas de l'échelle et qui n'ont d'autre voie de survie dans le présent contexte de leur exclusion forcée.

Si c'est le prix qu'il faut collectivement payer pour "Faire du Québec le Japon nord-américain ! " (Gagné, 1987; dans Fortin, $1988: 60-61)$, il importe d'ores et déjà de reconnaître que la répartition des coûts sociaux et économiques exigés par ce projet de société aux horizons plus qu'incertains est parfaitement inégale. Si cette réalité a un sens, elle a aussi un nom : I'injustice sociale... ! Voilà pourquoi elle exige d'être dénoncée et commande une solidarité avec tous ceux et celles qui la subissent. Une solidarité qui, dans le concret, doit suivre la voie d'une alliance nécessaire avec les forces de résistance qui sont en même temps porteuses de changement, d'une forme de transformation sociale possible aux visées forcément radicales. 


\section{ANNEXE 1}

Barème du programme SOUTIEN FINANCIER 1989-1990

\begin{tabular}{|c|c|c|c|c|}
\hline & Adulte(s) & $\begin{array}{l}\text { Enfant(s) } \\
\text { à charge }\end{array}$ & Barème & $\begin{array}{c}\text { Revenus de } \\
\text { travail } \\
\text { permis }^{1}\end{array}$ \\
\hline 1989 & 1 & 0 & $526 \$$ & $100 \$$ \\
\hline $1^{\text {er }}$ août - & 1 & 1 & $713 \$$ & $100 \$$ \\
\hline \multirow[t]{4}{*}{31 décembre } & 1 & 2 et plus & $778 \$$ & $100 \$$ \\
\hline & 2 & 0 & $820 \$$ & $100 \$$ \\
\hline & 2 & 1 & $894 \$$ & $100 \$$ \\
\hline & 2 & 2 et plus & $951 \$$ & $100 \$$ \\
\hline 1990 & 1 & 0 & $545 \$$ & $100 \$$ \\
\hline $\boldsymbol{1}^{\mathrm{er}}$ janvier - & 1 & 1 & $736 \$$ & $100 \$$ \\
\hline \multirow[t]{4}{*}{31 mars } & 1 & 2 et plus & $811 \$$ & $100 \$$ \\
\hline & 2 & 0 & $834 \$$ & $100 \$$ \\
\hline & 2 & 1 & $918 \$$ & $100 \$$ \\
\hline & 2 & 2 et plus & $980 \$$ & $100 \$$ \\
\hline 1990 & 1 & 0 & $578 \$$ & $100 \$$ \\
\hline $1^{\text {er }}$ avril - & 1 & 1 & $778 \$$ & $100 \$$ \\
\hline \multirow[t]{4}{*}{31 juillet } & 1 & 2 et plus & $869 \$$ & $100 \$$ \\
\hline & 2 & 0 & $858 \$$ & $100 \$$ \\
\hline & 2 & 1 & $961 \$$ & $100 \$$ \\
\hline & 2 & 2 et plus & $1032 \$$ & $100 \$$ \\
\hline
\end{tabular}

1. Les revenus de travail permis doivent provenir d'un travail ou d'une allocation de formation professionnelle. 


\section{ANNEXE 2}

\section{Barème du programme APTE 1989}

\begin{tabular}{|c|c|c|c|c|}
\hline & Adute(s) & $\begin{array}{l}\text { Enfant(s) } \\
\text { à charge }\end{array}$ & Barème & $\begin{array}{c}\text { Revenus de } \\
\text { travail } \\
\text { permis }^{1}\end{array}$ \\
\hline \multirow[t]{6}{*}{ Disponible } & 1 & 0 & $487 \$$ & $80 \$$ \\
\hline & 1 & 1 & $680 \$$ & $80 \$$ \\
\hline & 1 & 2 et plus & $782 \$$ & $80 \$$ \\
\hline & 2 & 0 & $770 \$$ & $50 \$$ \\
\hline & 2 & 1 & $885 \$$ & $50 \$$ \\
\hline & 2 & 2 et plus & $962 \$$ & $50 \$$ \\
\hline \multirow[t]{6}{*}{ Non disponible } & 1 & 0 & $507 \$$ & $53 \$$ \\
\hline & 1 & 1 & $690 \$$ & $70 \$$ \\
\hline & 1 & 2 et plus & $792 \$$ & $70 \$$ \\
\hline & 2 & 0 & $806 \$$ & $40 \$$ \\
\hline & 2 & 1 & $870 \$$ & $65 \$$ \\
\hline & 2 & 2 et plus & $942 \$$ & $70 \$$ \\
\hline \multirow[t]{6}{*}{ Participant } & 1 & 0 & $520 \$$ & $80 \$$ \\
\hline & 1 & 1 & $720 \$$ & $80 \$$ \\
\hline & 1 & 2 et plus & $822 \$$ & $80 \$$ \\
\hline & 2 & 0 & $850 \$$ & $50 \$$ \\
\hline & 2 & 1 & $965 \$$ & $50 \$$ \\
\hline & 2 & 2 et plus & $1042 \$$ & $50 \$$ \\
\hline \multirow[t]{6}{*}{ Non participant } & 1 & 0 & $420 \$$ & $140 \$$ \\
\hline & 1 & 1 & $620 \$$ & $140 \$$ \\
\hline & 1 & 2 et plus & $722 \$$ & $140 \$$ \\
\hline & 2 & 0 & $650 \$$ & $170 \$$ \\
\hline & 2 & 1 & $765 \$$ & $170 \$$ \\
\hline & 2 & 2 et plus & $842 \$$ & $170 \$$ \\
\hline
\end{tabular}

1. Les revenus de travail permis doivent provenir d'un travail ou d'une allocation de formation professionnelle. 
ANNEXE 3

\section{Barème mixte APTE 1989}

\begin{tabular}{llrr}
\hline Adulte(s) & $\begin{array}{c}\text { Enfant(s) } \\
\text { à charge }\end{array}$ & Barème & $\begin{array}{c}\text { Revenus de } \\
\text { travail } \\
\text { permis }\end{array}$ \\
\hline 1 non participant et & 0 & $750 \$$ & $110 \$$ \\
1 participant & 1 & $865 \$$ & $110 \$$ \\
& 2 et plus & $942 \$$ & $110 \$$ \\
1 non participant et & 0 & $728 \$$ & $92 \$$ \\
1 non disponible & 1 & $818 \$$ & $117 \$$ \\
& 2 et plus & $892 \$$ & $120 \$$ \\
1 non disponible et & 0 & $828 \$$ & $40 \$$ \\
1 participant & 1 & $918 \$$ & $57 \$$ \\
& 2 et plus & $992 \$$ & $60 \$$ \\
1 disponible et & 0 & $710 \$$ & $110 \$$ \\
1 non participant & 1 & $825 \$$ & $110 \$$ \\
& 2 et plus & $902 \$$ & $110 \$$ \\
1 disponible et & 0 & $788 \$$ & $40 \$$ \\
1 non disponible & 1 & $878 \$$ & $57 \$$ \\
& 2 et plus & $952 \$$ & $60 \$$ \\
1 disponible et & 0 & $810 \$$ & $50 \$$ \\
& 1 & $925 \$$ & $50 \$$ \\
\hline participant & & $1002 \$$ & $50 \$$ \\
\hline
\end{tabular}

1. Les revenus de travail permis doivent provenir d'un travail ou d'une allocation de formation professionnelle.

\section{Notes}

1 À ce propos, voir la communication présentée au Colloque tenu à Montréal en septembre 1989 sur le thème "Santé mentale et pauvreté : un silence troublant" (Fortin, 1989). Voir également le document présenté à la Conférence internationale de Berlin du Conseil international de l'action sociale (CIAS, 1988) par Denis Fortin.

2 Par rapport à cette question, les " nouvelles " intentions du ministre de la Main-d'œuvre et de la Sécurité du revenu, André Bourbeau, n'ont rien de rassurant, il s'en faut ! Voir Boivin (1989). 
${ }^{3}$ Pour plus d'informations à cet égard, se référer aux sources suivantes :

- Loi sur la sécurité du revenu (loi 37) sanctionnée le 22 décembre 1988, Éditeur officiel du Québec, 44 pages.

- Règlement sur la sécurité du revenu, Décret 922-89, 14 juin 1989, extrait de la Gazette officielle du Québec, p. 3304-3325.

- La sécurité du revenu. En bref, Ministère de la Main-d'œuvre et de la Sécurité du revenu, 1989, 30 pages.

- La sécurité du revenu, feuillet explicatif et d'information, Ministère de la Main-d'œuvre et de la Sécurité du revenu, 1989, 40 pages.

${ }^{4} \mathrm{C}^{\prime}$ est dans le cadre d'une intervention faite sur le thème " Travail précaire et chômage chronique ", au Forum national pour l'emploi tenu à Montréal en novembre 1989, que nous avons présenté notre position par rapport à cette question, de même que les données empiriques principales à la base de celle-ci. Pour qui veut en approfondir le contenu, les quelques références suivantes seront utiles : Fortin (1988: 105-124); Rabeau (1989 : 166-172); article dans Les Affaires, 18 novembre 1989:25 (auteur non identifié) : "Recherche d'un emploi : les travailleurs québécois se découragent toujours autant "; Roy (1989); Ministère de la Main-d'œuvre et de la Sécurité du revenu (1989).

\section{Références}

BoIvin, G. (1989). "Aide sociale : les "bouboumacoutes" auront encore plus de pouvoir en 1990 ", Le Soleil, 15 décembre 1989 : A-5.

Conseil international de I'action sociale (CIAS) (1988). Crise et politique sociale. Ou l'oppression... ! (Rapport du Groupe-Canada du CIAS).

FORTIN, D. (1988). Riches contre pauvres. Deux poids, deux mesures !. Éditions Autogestionnaires.

ForTIN, D. (1989). Vivre l'humiliation... ou la réforme de l'aide sociale au Québec! Communication présentée au Colloque "Santé mentale et pauvreté : un silence troublant», en septembre 1989.

GAGNÉ, J.P. (1987). "MacDonald aux Affaires : Faire du Québec le Japon nordaméricain ", Les Affaires, 24-30 janvier $1987: 1$-3.

Ministère de la Main-d'œuvre et de la Sécurité du revenu (1989). La maind'œuvre, l'emploi et les disparités régionales au Québec.

RABEAU, Y. (1989). "Chômage : nous pourrions faire mieux", Commerce, octobre : 166-172.

RoY, A. (1989). "Beaucoup d'emplois de piètre qualité au Canada depùis I'arrivée des conservateurs", Le Soleil, 4 juin : A-6. 